K. Shiraiwa

Nagoya Math. J,

Vol. 49 (1973), 111-115

\title{
MANIFOLDS WHICH DO NOT ADMIT ANOSOV DIFFEOMORPHISMS
}

\author{
KENICHI SHIRAIWA
}

In [3], M. W. Hirsch obtained some necessary conditions for the existence of an Anosov diffeomorphism on a differentiable manifold. As an application, he constructed many manifolds which do not admit Anosov diffeomorphisms.

In this paper, we shall give a new necessary condition for the existence of an Anosov diffeomorphism on a compact connected differentiable manifold. Our result seems weaker than that of M. W. Hirsch's, but it can be applied for the case in which his results are not applicable. For example, the following manifolds are among those which do not admit Anosov diffeomorphisms: Homotopy spheres, projective spaces over real, complex, and quaternion number fields, and lens spaces.

Throughout this paper "differentiable" means "differentiable of class $C^{\infty}$ " unless otherwise stated.

\section{§1. The Main Theorem}

Let $M$ be a Riemannian manifold. A $C^{1}$-diffeomorphism $f: M \rightarrow M$ is called an Anosov diffeomorphism if there exist a continuous splitting

$$
T(M)=E^{u} \oplus E^{s}
$$

of the tangent bundle of $M$ and constants $C>0, \lambda>1$ such that $T f\left(E^{u}\right)$ $=E^{u}, T f\left(E^{s}\right)=E^{s}$, and, furthermore, for all positive integers $m$ and tangent vectors $x \in T(M)$ :

$$
\begin{array}{ll}
\left|T f^{m}(x)\right| \geqq C \lambda^{m}|x| & \text { if } x \in E^{u}, \\
\left|T f^{m}(x)\right| \leqq C^{-1} \lambda^{-m}|x| & \text { if } x \in E^{s},
\end{array}
$$

where $T f^{m}$ is the tangent of $f^{m}$, and $|\cdot|$ is the norm of the tangent vector. 
If $M$ is compact, then this condition is independent of the Riemannian metric on $M$.

For a diffeomorphism $f: M \rightarrow M$, we denote the set of fixed points (resp. periodic points) of $f$ by $\operatorname{Fix}(f)$ (resp. Per $(f)$ ). Also, the set of non-wandering points is denoted by $\Omega(f)$. Then,

$$
\text { Fix }(f) \subset \operatorname{Per}(f) \subset \Omega(f) \text {. }
$$

THEOREM 1. Let $M$ be a compact connected differentiable manifold without boundary. If $f: M \rightarrow M$ is an Anosov diffeomorphism, then the induced homomorphism $f_{*}: H_{*}(M ; Q) \rightarrow H_{*}(M ; Q)$ is not the identity map, where $H_{*}(M ; Q)$ is the homology of $M$ with coefficients in the field of rational numbers $\boldsymbol{Q}$.

Proof. Let $f: M \rightarrow M$ be an Anosov diffeomorphism. Then any iterate $f^{n}(n \neq 0)$ of $f$ is an Anosov diffeomorphism. Therefore, any fixed points of $f^{n}$ are hyperbolic fixed points and, hence, isolated. Since all the fixed points of an Anosov diffeomorphism have the same index \pm 1 , the absolute value of the Lefschetz number $L\left(f^{n}\right)$ is equal to the number of the fixed points of $f^{n}$. Thus, we obtain

(1) the number of the elements of $\operatorname{Fix}\left(f^{n}\right)$ is equal to $\left|L\left(f^{n}\right)\right|$.

Since any fixed point of $f$ is a fixed point of $f^{n}$, we obtain

$$
\operatorname{Fix}(f) \subset \operatorname{Fix}\left(f^{n}\right) \quad \text { for each } n \in \boldsymbol{Z} \text {, }
$$

where $Z$ is the group of integers.

Now assume that $f_{*}: H_{*}(M ; Q) \rightarrow H_{*}(M ; Q)$ is the identity map. Then, any iterate $f^{n}$ of $f$ induces the identity map on $H_{*}(M ; Q)$. Therefore,

$$
L\left(f^{n}\right)=L(i d .)=\chi(M) \quad \text { for each } n \in Z,
$$

where $\chi(M)$ is the Euler characteristic of $M$.

By (1), (2), (3), we have

$$
\operatorname{Fix}(f)=\operatorname{Fix}\left(f^{n}\right) .
$$

Since $\operatorname{Per}(f)=\bigcup_{n \geqq 1} \operatorname{Fix}\left(f^{n}\right)$, we have

$$
\operatorname{Per}(f)=\operatorname{Fix}(f) \text {, and } \operatorname{Per}(f) \text { is finite. }
$$

Together with J. Frank's result [2] that $\operatorname{Per}(f)$ is dense in $\Omega(f)$ for an Anosov diffeomorphism $f$, we have 
$\Omega(f)=\overline{\operatorname{Per}(f)}=\operatorname{Per}(f)=\operatorname{Fix}(f)$, and $\Omega(f)$ is a finite set consisting of the hyperbolic fixed points of $f$.

It is also known that the continuous splitting $T(M)=E^{u} \oplus E^{s}$ of the tangent bundle of $M$ defines a foliation on $M$, and the stable and unstable manifolds are the leaves of this foliation. Thus, for each $p, q \in \Omega(f)=$ $\operatorname{Per}(f)$, the stable manifold $W^{s}(p)$ and the unstable manifold $W^{u}(q)$ have transversal intersection (Cf. [4]). Thus, $f$ is a Morse-Smale diffeomorphism and, hence, the Morse-Smale inequalities hold (Cf. [4]).

Since $\operatorname{dim} H_{0}(M, Q)=1$, there exists a point $p \in \Omega(f)$ such that $\operatorname{dim} W^{s}(p)=0$. Dually, there exists a point $q \in \Omega(f)$ such that $\operatorname{dim} W^{u}(q)$ $=0$, i.e., $\operatorname{dim} W^{s}(q)=\operatorname{dim} M$. But, since $W^{s}(p)$ and $W^{s}(q)$ are leaves of the foliation $T(M)=E^{u} \oplus E^{s}$, and since $M$ is connected, $\operatorname{dim} W^{s}(p)=$ $\operatorname{dim} W^{s}(q)=\operatorname{dim} E^{s}$. This is a contradiction. Therefore, $f_{*}: H_{*}(M, Q)$ $\rightarrow H_{*}(M, Q)$ is not the identity map.

CoRollary 1. Let $M$ be a compact connected differentiable manifold without boundary. If $f: M \rightarrow M$ is an Anosov diffeomorphism, then any iterate $f^{n}(n \neq 0)$ of $f$ does not induce the identity map on $H_{*}(M ; Q)$.

Proof. If $f$ is an Anosov diffeomorphism, $f^{n}(n \neq 0)$ is also an Anosov diffeomorphism. Therefore, $f_{*}^{n}$ is not the identity map on $H_{*}(M ; Q)$.

CoROLlary 2. Let $M$ be a compact differentiable manifold of dimension $m$. If $M$ is a rational homology $m$-sphere, i.e., the homology of $M$ with eoefficients in $\boldsymbol{Q}$ is isomorphic to that of the $m$ dimensional sphere $S^{m}$, then $M$ does not admit an Anosov diffeomorphism. In particular, any homotopy sphere does not admit an Anosov diffeomorphism.

Proof. Assume that $M$ admits an Anosov diffeomorphism $f: M \rightarrow M$. Then $f^{2}: M \rightarrow M$ is an orientation preserving diffeomorphism. Therefore, $f^{2}$ induces the identity map on $H_{*}(M ; Q)$. This contradicts Corollary 1.

COROLlaRY 3. Let $M$ be a differentiable manifold such that its universal covering space is a rational homology sphere. Then $M$ does not admit an Anosov diffeomorphism. In particular, the lens spaces and real projective spaces do not admit Anosov diffeomorphisms.

Proof. Assume that $M$ admit an Anosov diffeomorphism $f: M \rightarrow M$. Let $\tilde{M}$ be a universal covering space of $M$ and let $p: M \rightarrow \tilde{M}$ be its projection. Then $f: M \rightarrow M$ can be lifted to a diffeomorphism $\tilde{f}: \tilde{M} \rightarrow \tilde{M}$. 
Using the Anosov structure of $f$ on $M$ and the projection $p: \tilde{M} \rightarrow M$, we can easily prove that $\tilde{f}$ is also an Anosov diffeomorphism. If $\tilde{M}$ is a rational homology sphere, this contradicts Corollary 2 .

\section{§ 2. Some Applications}

Let $X$ be a finite $C W$-complex. The set $\mathscr{E}(X)$ of all the homotopy classes of the homotopy equivalences of $X$ into itself forms a group under the composition operation. The homotopy class of $f: X \rightarrow X$ is denoted by $[f]$.

For each $\alpha=[f] \in \mathscr{E}(X)$, we associate the induced automorphism $f_{*}: H_{*}(X ; \boldsymbol{Z}) \rightarrow H_{*}(X ; \boldsymbol{Z})$. This is independent of the choice of a representative $f$ of $\alpha$. Hence it defines a homomorphism $H: \mathscr{E}(X) \rightarrow$ Aut $\left(H_{*}(X ; \boldsymbol{Z})\right)$, where Aut $\left(H_{*}(X ; \boldsymbol{Z})\right)$ is the group of the automorphisms of $H_{*}(X ; Z)$ onto itself as a graded group.

The kernel $\mathscr{E}_{0}(X)$ of $H$ consists of the homotopy classes of the homotopy equivalences $f: X \rightarrow X$ such that $f_{*}: H_{*}(X ; Z) \rightarrow H_{*}(X ; Z)$ is the identity map.

Theorem 2. Let $M$ be a compact connected differentiable manifold without boundary such that $\mathscr{E}(M) / \mathscr{E}_{0}(M)$ is finite. Then $M$ does not admit an Anosov diffeomorphism. In particular, if $\mathscr{E}(M)$ is finite, the same holds.

Proof. If $\mathscr{E}(M) / \mathscr{E}_{0}(M)$ is finite, then for any diffeomorphism $f: M \rightarrow M$ there exists a positive integer $n$ such that $\left[f^{n}\right] \in \mathscr{E}_{0}(M)$. Thus $f^{n}$ induces the identity map on $H_{*}(M ; \boldsymbol{Z})$. Therefore, $f_{*}^{n}: H_{*}(M ; \boldsymbol{Q}) \rightarrow H_{*}(M ; \boldsymbol{Q})$ is also the identity map. Corollary 1 to Theorem 1 implies that $f$ is not an Anosov diffeomorphism.

COROLLARY 1. Let $M$ be a compact connected differentiable manifold without boundary such that $\operatorname{Aut}\left(H_{*}(M ; Z)\right)$ is finite. Then $M$ does not admit an Anosov diffeomorphism.

Proof. Since $H$ induces an isomorphism of $\mathscr{E}(M) / \mathscr{E}_{0}(M)$ onto a subgroup of $\operatorname{Aut}\left(H_{*}(M ; Z)\right), \mathscr{E}(M) / \mathscr{E}_{0}(M)$ is finite if $\operatorname{Aut}\left(H_{*}(M ; Z)\right)$ is finite.

COROLLARY 2. Let $M$ be a compact connected differentiable manifold without boundary. If $\operatorname{dim} H_{k}(M ; Q) \leqq 1$ for each $k \geqq 0$, then $M$ does not admit an Anosov diffeomorphism. In particular, real, complex, and 
quaternion projective spaces and Cayley projective plane do not admit Anosov diffeomorphisms.

Proof. Let $F_{k}$ be the torsion subgroup of $H_{k}(M ; Z)$. Then, there exists an exact sequence

$$
0 \longrightarrow F_{k} \longrightarrow H_{k}(M ; Z) \longrightarrow G_{k} \longrightarrow 0,
$$

where $G_{k}$ is a free abelian group with $\operatorname{dim} H_{k}(M ; Q)$ generators. Any automorphism of $H_{k}(M ; Z)$ induces an automorphism of the above exact sequence. If $\operatorname{dim} H_{k}(M ; Q) \leqq 1$, then the number of the automorphisms of $G_{k}$ is at most two. Therefore, $\operatorname{Aut}\left(H_{*}(M ; Z)\right)$ is finite if $\operatorname{dim} H_{k}(M, Q)$ $\leqq 1$ for each $k \geqq 0$.

COROLlaRY 3. Let $M$ be a simply connected compact differentiable manifold without boundary. If its rational cohomology ring is isomorphic to that of a product of odd dimensional spheres of distinct dimensions, then $M$ does not admit an Anosov diffeomorphism.

Proof. By Corollary 5.5 of M. Arkowitz-C. R. Curjel [1], $\mathscr{E}(M)$ is finite. Thus Theorem 2 implies the result.

The following are among the manifolds which satisfy the conditions of Corollary 3 and hence do not admit Anosov diffeomorphisms: Spin $(2 n-1), S U(n), S p(n)$, and the simply connected exceptional Lie groups, i.e., $G_{2}, F_{4}, E_{6}, E_{7}, E_{8}$.

\section{REFERENCES}

[1] M. Arkowitz-C. R. Curjel: Groups of Homotopy Classes, Lecture Notes in Mathematics, Springer Verlag, Berlin (1964).

[ 2 ] J. Franks: Anosov Diffeomorphisms, Proc. Symp. Pure Mathematics XIV, Global Analysis, Amer. Math. Soc., Province, R.I. (1970).

[ 3 ] M. W. Hirsch: Anosov Maps, Polycyclic Groups and Homology, Topology 10 (1971), 177-183.

[ 4 ] S. Smale: Differentiable Dynamical Systems, Bull. Amer. Math. Soc. 73 (1967), $747-817$.

Nagoya University 